\title{
An Analysis of the Solar Rotation Velocity by Tracing Coronal Features
}

\author{
R. Brajša*, B. Vršnak, V. Ruždjak \\ Hvar Observatory, Faculty of Geodesy, University of Zagreb, P. O. B. \\ 164, HR-10001 Zagreb, Croatia \\ D. Roša, D. Hržina \\ Astronomical Observatory, Astronomical Association of Zagreb, \\ Opatička 22, HR-10000 Zagreb, Croatia \\ H. Wöhl \\ Kiepenheuer-Institut für Sonnenphysik, Schöneckstr. 6, D-79104 \\ Freiburg, Germany \\ F. Clette, J.-F. Hochedez \\ Observatoire Royal de Belgique, Departement de Physique Solaire, \\ Avenue Circulaire 3, B-1180 Bruxelles, Belgique
}

\begin{abstract}
The solar rotation is determined tracing coronal EUV and soft X-ray bright points, active regions, parts of coronal holes and foot points of coronal loops. Full-disc solar images in the EUV part of the spectrum from the $S O H O$ spacecraft (EIT, Fe XV, $28.4 \mathrm{~nm}$ ) and from the Yohkoh satellite (SXT, $2 \mathrm{~nm}$ ) are used.
\end{abstract}

\section{Introduction}

Full-disc solar images from the Yohkoh and SOHO missions (Golub and Pasachoff 1997) give an unique opportunity to study the solar rotation tracing features in EUV and X-ray parts of the spectrum. In this work we have traced several types of coronal features, primarily coronal bright points, which may represent very appropriate tracers for the solar rotation analysis because of their small diameters, large number and presence at many latitudes simultaneously.

\section{The SOHO-EIT Data Set, Reduction Method and Results}

Full-disc EUV solar images in $\mathrm{Fe} \mathrm{XV}$ at the wavelength of $28.4 \mathrm{~nm}$ from the SOHO-EIT were used. Our data set consists of about 600 full-disc filtergrams obtained most often with a cadence of 4 images per 24 hours and mainly equally

\footnotetext{
*Alexander von Humboldt Research Fellow; present address: Kiepenheuer-Institut für Sonnenphysik, Schöneckstr. 6, D-79104 Freiburg, Germany
} 


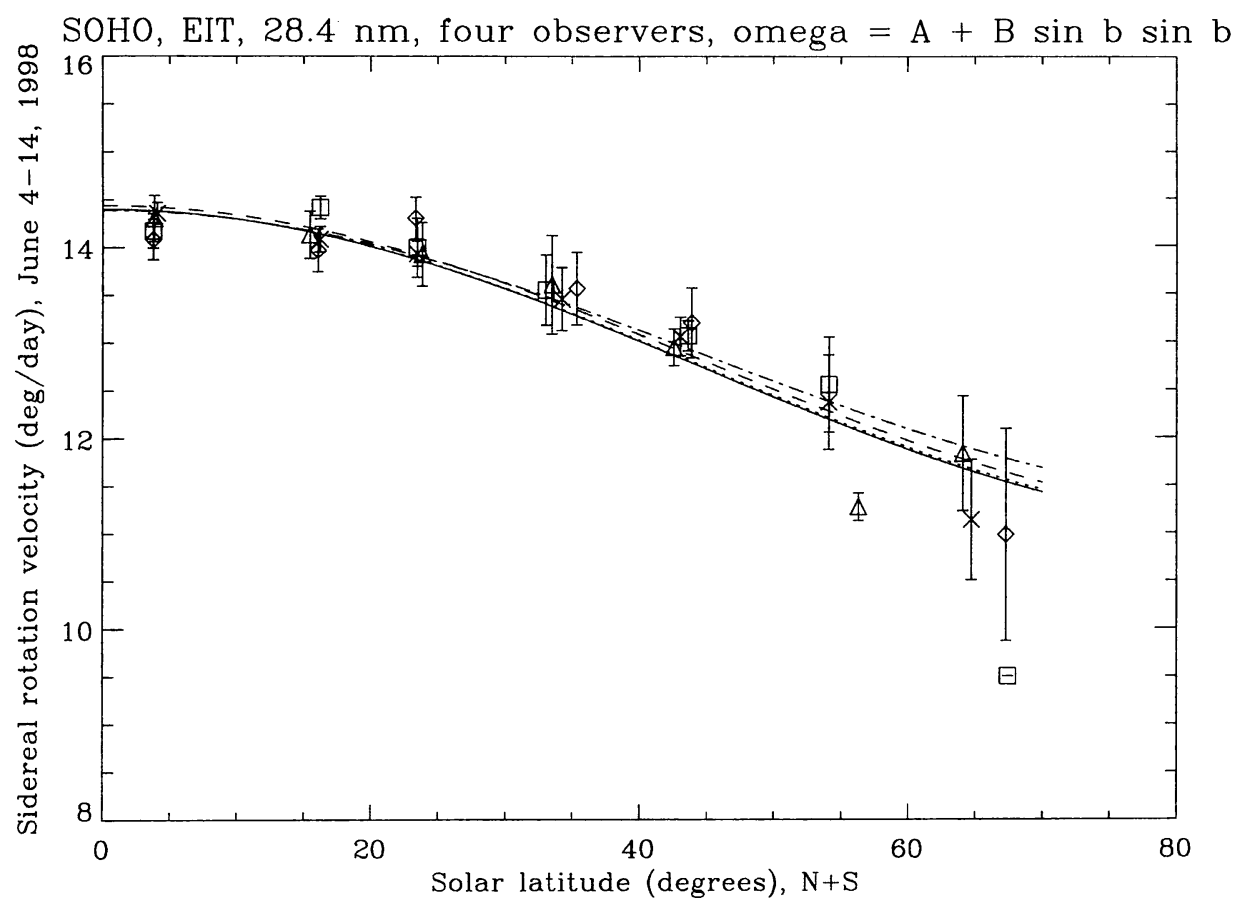

Figure 1. Mean values of solar sidereal angular rotation velocities obtained tracing coronal bright points in both solar hemispheres during June 4-14, 1998 by four observers are presented by four different symbols. Measurements were performed in $10 \mathrm{deg}$ latitude bands and the error bars indicate standard errors of the means in each bin. The four lines represent fits to all data points of each of the four observer.

distributed in time. In this work the solar rotation was analysed tracing coronal EUV bright points in the first 40 images from our data set in the time interval June 4-14, 1998 by four different observers independently. The reduction method is described in detail by Brajša et al. (2000).

Mean values of solar sidereal angular rotation velocities for 10 deg latitude bands obtained by four observers are presented in Figure 1 by series of four different symbols. The solar differential rotation velocity is represented by

$$
\omega(b)=A+B \sin ^{2} b,
$$

where $\omega$ is the sidereal angular rotation velocity in $\mathrm{deg} /$ day, $b$ the heliographic latitude, and $A, B$ are the solar differential rotation velocity parameters. The four different curves in Figure 1 were obtained with parameters from the expression (1) for the results of the four different observers. In each case, all data points without any filtering were used in the fitting procedures. The number of identified coronal bright points was different for the four observers, having the minimal value of 66 and the maximal value of 208 bright points which were traced and whose rotation velocity was determined. 


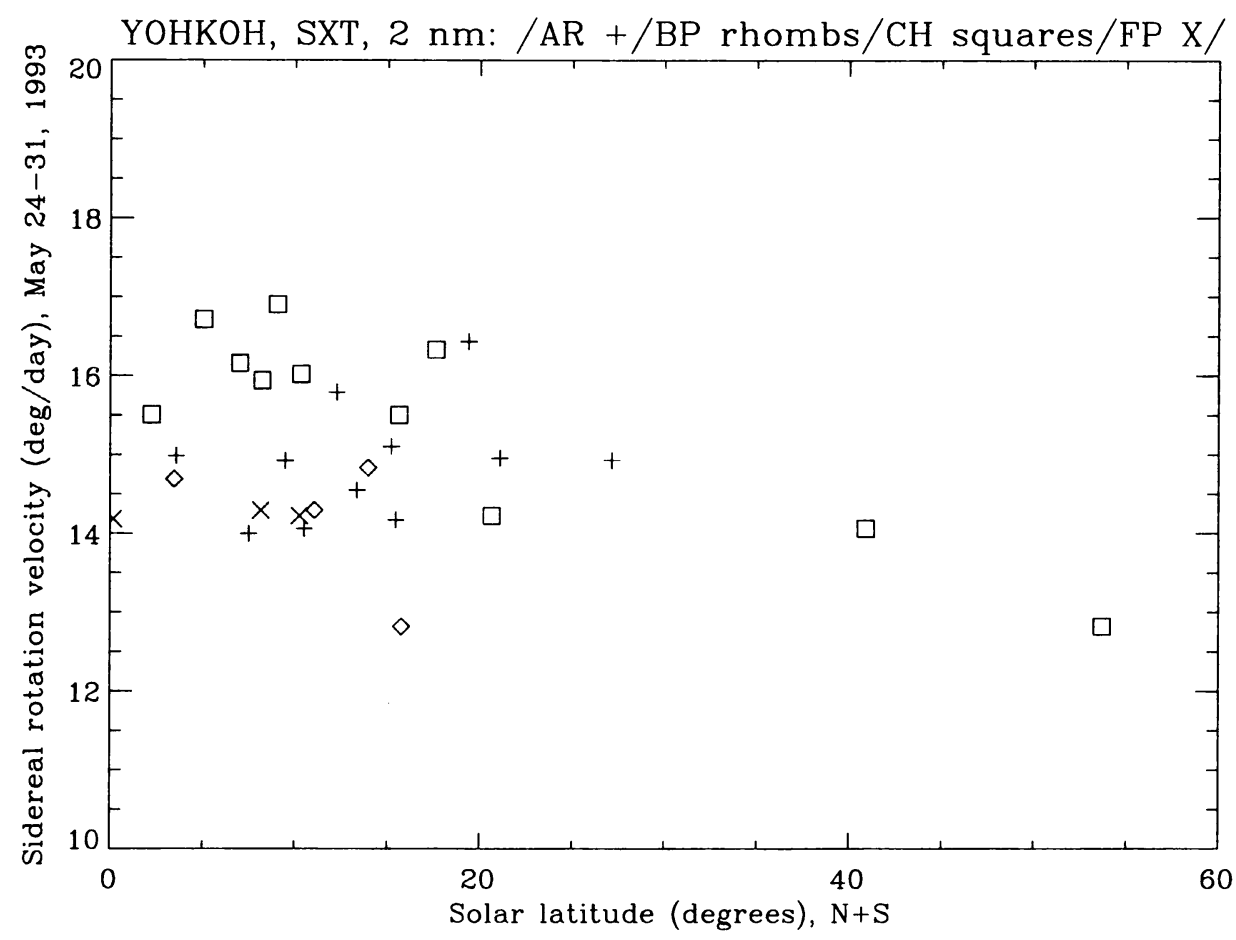

Figure 2. Solar sidereal angular rotation velocities obtained tracing different coronal structures during May 24-31, 1993: active regions = + , bright points $=\diamond$, coronal holes $=\square$, foot points of loops $=x$.

\section{The Yohkoh-SXT Data Set, Reduction Method and Results}

Daily full-disc solar images in soft X-rays from the Yohkoh-SXT (Al/Mg filter transmitting at the wavelength of about $2 \mathrm{~nm}$ ) were analysed and various coronal structures identified to determine the solar rotation velocity. In total, 29 structures were traced during May 24-31, 1993, among them active regions, bright points, parts of coronal holes and foot points of coronal loops. The results are presented in Figure 2, where different symbols indicate rotation velocities of different coronal structures. Please note that both axes have different scales in Figures 1 and 2.

\section{Discussion and Conclusion}

Let us firstly discuss the results on the solar rotation obtained tracing coronal EUV bright points. There is no significant difference among the solar rotation velocity values measured by the four observers (Figure 1). This implies that the results are independent from the subjective differences which may occur among the observers, in spite of the fact that very different numbers of EUV bright points were traced in the same time period. The average solar differential 
rotation profile can be described by the mean values of the four sets of rotation parameters from expression (1):

$$
\omega(b)=14.41-3.27 \sin ^{2} b .
$$

The mean equatorial sidereal rotation velocity $(14.41 \mathrm{deg} / \mathrm{day})$ from equation (2) is somewhat lower than the equatorial sidereal rotation velocity previously determined tracing EUV bright points (14.65 $\pm 0.2 \mathrm{deg} /$ day) and X-ray bright points (14.6 $\pm 0.3 \mathrm{deg} /$ day) in other studies (Dupree and Henze 1972; Simon and Noyes 1972; Golub et al. 1974).

In Figure 2 sidereal rotation velocities obtained tracing various coronal structures in soft X-rays are presented. There are less velocity measurements in this case than for the EUV bright points and so the results here are less accurate. However, three of the four rotation velocities of coronal soft X-ray bright points in the latitude range 0-20 deg have values between 14 and $15 \mathrm{deg} /$ day (Figure 2 ) in a good agreement with the velocity values obtained tracing EUV bright points (Figure 1).

We can thus already conclude that coronal bright points represent quite good tracers for the determination of the solar rotation. However, in this paper only some very preliminary results are presented and further data reductions are necessary, especially containing data from various phases of the solar cycle. This can be performed since both missions, Yohkoh and SOHO are covering many subsequent years.

Acknowledgments. This work was undertaken with the support of the Alexander von Humboldt Foundation and is related to the SOHO-EIT Proposal Brajsa_206 and to the Yohkoh-SXT Proposal qs061.sakurai04. SOHO is a project of international cooperation between ESA and NASA. We would like to thank Dr. T. Sakurai who provided full-disc solar images from the Yohkoh-SXT and the students M. Kasabašić and J. Rodmann who have taken part in the data reduction.

\section{References}

Brajša, R., Wöhl, H., Kasabašić, M., Rodmann, J., Vršnak, B., Ruždjak, V., Roša, D., Hržina, D., Clette, F., and Hochedez, J.-F. 2000, Hvar Obs. Bull., 24, in press

Dupree, A. K. and Henze, W., Jr. 1972, Solar Phys., 27, 271

Golub, L. and Pasachoff, J. M. 1997, The Solar Corona, (Cambridge: Cambridge University Press)

Golub, L., Krieger, A. S., Silk, J. K., Timothy, A. F., and Vaiana, G. S. 1974, ApJ, 189, L93

Simon, G. W. and Noyes, R. W. 1972, Solar Phys., 22, 450 